\title{
Conductance distribution near the Anderson transition
}

\author{
I. M. Suslov \\ P.L.Kapitza Institute for Physical Problems, \\ 119334 Moscow, Russia \\ E-mail: suslov@kapitza.ras.ru
}

\begin{abstract}
Using a modification of the Shapiro approach, we introduce the two-parameter family of conductance distributions $W(g)$, defined by simple differential equations, which are in the one-to-one correspondence with conductance distributions for quasi-one-dimensional systems of size $L^{d-1} \times L_{z}$, characterizing by parameters $L / \xi$ and $L_{z} / L$ ( $\xi$ is the correlation length, $d$ is the dimension of space). This family contains the Gaussian and log-normal distributions, typical for the metallic and localized phases. For a certain choice of parameters, we reproduce the results for the cumulants of conductance in the space dimension $d=2+\epsilon$ obtained in the framework of the $\sigma$-model approach. The universal property of distributions is existence of two asymptotic regimes, log-normal for small $g$ and exponential for large $g$. In the metallic phase they refer to remote tails, in the critical region they determine practically all distribution, in the localized phase the former asymptotics forces out the latter. A singularity at $g=1$, discovered in numerical experiments, is admissible in the framework of their calculational scheme, but related with a deficient definition of conductance. Apart of this singularity, the critical distribution for $d=3$ is well described by the present theory. One-parameter scaling for the whole distribution takes place under condition, that two independent parameters characterizing this distribution are functions of the ratio $L / \xi$.
\end{abstract}

\section{Introduction}

Dimensionless conductance $g=h G / e^{2}$ is determined by conductance $G=\sigma L^{d-2}$ of a system in quantum units $e^{2} / h$; the system is supposed to have a form of the $d$-dimensional cube with a side $L$, and $\sigma$ is conductivity. One of the fundamental problems in theory of disordered systems is related with the conductance distribution $W(g)$ [1]-18. Its actuality was realized after discovery of the so called "universal conductance fluctuations" in the metallic state 1, 2,

$$
\left\langle(\delta g)^{2}\right\rangle=c \sim 1
$$

where the constant $c$ in the right-hand-side does not depend on the system size and the strength of disorder, but depends on the dimension of space and the boundary conditions. Near the Anderson transition the average value $\langle g\rangle$ is also of the order of unity, so conductance is a strongly fluctuating quantity and may be not adequately described by its first moment. Investigation of higher moments in the framework of the sigma-model approach [3, 4, leads to the following results for the cumulants of conductance 11 at the

\footnotetext{
${ }^{1}$ Recall that the characteristic function $F(t)=$ $\left\langle e^{i g t}\right\rangle$ is the generating function for moments $(F(t)=$ $\left.\sum_{n=0}^{\infty}(i t)^{n}\left\langle g^{n}\right\rangle / n !\right)$, while its logarithm is the generating function of cumulants $\left(\ln F(t)=\sum_{n=0}^{\infty}(i t)^{n}\left\langle\left\langle g^{n}\right\rangle\right\rangle / n\right.$ !). In particular, the second cumulant is a dispersion of the distribution.
}

transition point for the space dimension $d=2+\epsilon$

$$
\left\langle\left\langle g^{n}\right\rangle\right\rangle \sim\left\{\begin{array}{cc}
\epsilon^{n-2}, & n<n_{0} \\
L^{\epsilon n^{2}-2 n}, & n>n_{0}
\end{array},\right.
$$

where $n_{0} \sim 1 / \epsilon$. The system size $L$ in the second relation is dimensionalized by a microscopic scale like the mean-free-path $l$ or the lattice spacing $a$. It led the authors of [3, 4, to conclusion on violation of the one-parameter scaling hypothesis [5], according to which $g$ is completely determined by the ratio $L / \xi$, where $\xi$ is the correlation length.

This conclusion was contested by Shapiro $6,7,8$, who argued that divergency of high moments may be determined by a negligible part of the distribution in its far tail, while the main part of the distribution may obey one-parameter scaling 2 . Using the approximate Migdal-Kadanov scaling transformation, Shapiro has obtained for the distribution $P(\rho)$ of dimensionless resistances $(\rho=1 / g)$ at the critical point

$$
P_{c}(\rho)=\operatorname{const}(\rho+1)^{-\alpha},
$$

where $\alpha=1 / \epsilon$ for $d=2+\epsilon[6$, 7]. If the analogous result was obtained for the conductance distribution $W(g)$, it would give explanation of (2): the moments

\footnotetext{
${ }^{2}$ Analogous arguments were put forward in [9], where solution of a certain hierarchical model led to the distribution $W(g)$ with the power-law tail obeying one-parameter scaling.
} 
with $n \gtrsim 1 / \epsilon$ do not exist in the infinite system, and diverge with $L$ in a finite one. Using the requrrent relations, analogous to those for moments of $P(\rho)$, Shapiro has constructed an example of the conductance distribution $W(g)$, possessing the properties (2) and well defined in the thermodynamic limit; in analogy with (3) it has the power-law behavior $W(g) \sim g^{-2 / \epsilon}$ for large $g$. In spite of the evident success, the latter result is questionable: extrapolation to $\epsilon \sim 1$ gives a power-law tail with the exponent of the order of unity, contradicting to all numerical experiments 11 .

The latter contradiction looks rather fundamental, since at first glance Eqs.2 unambiguously indicate the power-law dependence with the exponent of the order $1 / \epsilon$. This puzzle is resolved in the present paper. We show that results (2) are not necessarily related with the power-law tail of the critical distribution and compatible with its exponential behavior at infinity; in this case, the first result (2) is valid for all cumulants. As for the second result (2), it corresponds to the situation when the critical distribution is subjected to perturbation $\delta W(g)$, retaining the system in the critical state 3 . Due to the normalization condition, a perturbation $\delta W(g)$ is necessarily alternating and its relaxation is described by the diffusion type equation. With the increase of the system size, $\delta W(g)$ undergoes a diffusion spreading and tends to zero in the limit $L \rightarrow \infty$; however, its tail extends to infinity and provides a divergency of high moments. The strange analogy between $P(\rho)$ and $W(g)$ discovered by Shapiro is also explained: these distributions are described by equations of the same structure in the space dimension $d=2+\epsilon$.

The main source of information on $W(g)$ are numerical experiments. At present, there is a common belief that the conductance distribution is Gaussian in the metallic state and log-normal in the localized phase, while at the critical point it is close to onesided log-normal [10] (Fig.1). More detailed investigation [11] shows (Fig.2), that the critical distribution can be divided into two parts: on the right of a certain point $A$ the logarithm of $W(g)$ is linear in $g$ (Fig.2,a), while on the left of $A$ the logarithm of $P(\ln g)$ is quadratic in $\ln g$ (Fig.2,b). According to Markos [11, point $A$ is a real singularity, which was confirmed by Muttalib et al 12 in the framework of a certain theoretical scheme. However, accord-

\footnotetext{
${ }^{3}$ In the renormalization group language [19] 20], it means that the system is deviated from the fixed point, but remains at the critical surface.
}

ing to the general principles of the modern theory of critical phenomena [19, 20], singularities are absent in finite systems and may arise only in the thermodynamic limit; it contradicts to the accepted stationarity of the critical distribution, which is formed at sufficiently small $L$ and then remains unchanged. Below we reproduce all listed properties and discuss the problem of a singularity.

Analysis of the present paper is based on a modification of the Shapiro approach 6, 7, 8, We introduce the two-parameter family of distributions, defined by simple differential equations, which are in the one-to-one correspondence with conductance distributions for quasi-one-dimensional systems of size $L^{d-1} \times L_{z}$, characterizing by parameters $L / \xi$ and $L_{z} / L$. This family contains the Gaussian and lognormal distributions, typical for the metallic and localized phases. For a certain choice of parameters, we reproduce results (2) for the cumulants in the space dimension $d=2+\epsilon$. The universal property of distributions is existence of two asymptotic regimes, log-normal for small $g$ and exponential for large $g$, while their actuality depends on the specific situation. In the metallic phase, a distribution is determined by the central Gaussian peak, while two asymptotic regimes refer to its far tails. In the critical region, the log-normal behavior is extended to a vicinity of the maximum, as we have seen in Fig.2, and practically all distribution is determined by two asymptotes. In proceeding to the localized phase, the log-normal behavior extends even more and forces out the exponential asymptotics to the region of the remote tail. A singularity at point $A$ is admissible in the framework of the calculational scheme used in [11, but related with a deficient definition of conductance; it will be smeared out for the correct definition. Apart of the latter moment, numerical data in Fig.2,b are well described by the present theory.

According to numerical experiments, oneparameter scaling is valid for the distribution $W(g)$ in whole. It is established by investigation of percentiles [13, and by analysis of average quantities $\langle g\rangle,\langle\rho\rangle,\langle\ln g\rangle$ 14. The present analysis is based on the assumption of one-parameter scaling and its results agree with this assumption. For a final decision on existence of scaling for the whole distribution, one should prove that two independent parameters, characterizing this distribution, (e.g., $\langle g\rangle$ and $\left.\left\langle(\delta g)^{2}\right\rangle\right)$ are functions only of the ratio $L / \xi$. For the first parameter, this property was 


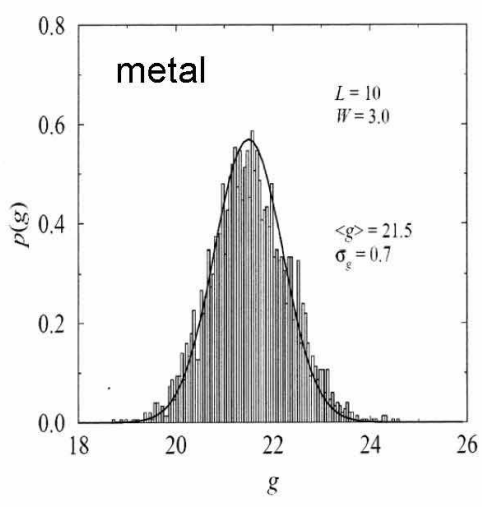

(a)

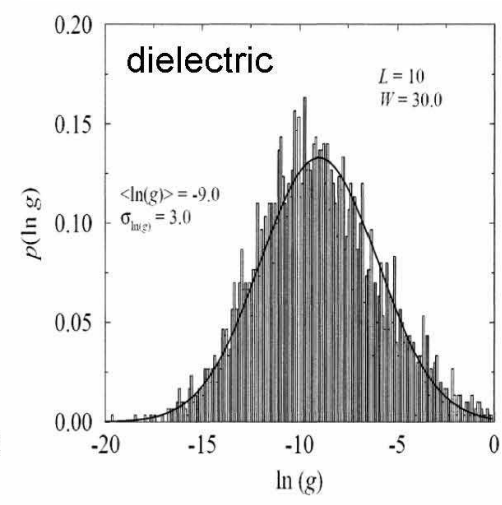

(b)

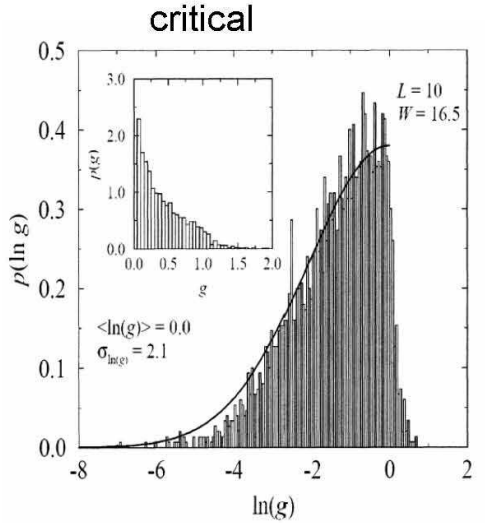

(c)

Figure 1: The conductance distribution is Gaussian in the metallic regime (a), log-normal in the localized phase (b), and close to one-sided log-normal at the critical point (c) (according to [10]). Solid lines correspond to the Gaussian law.

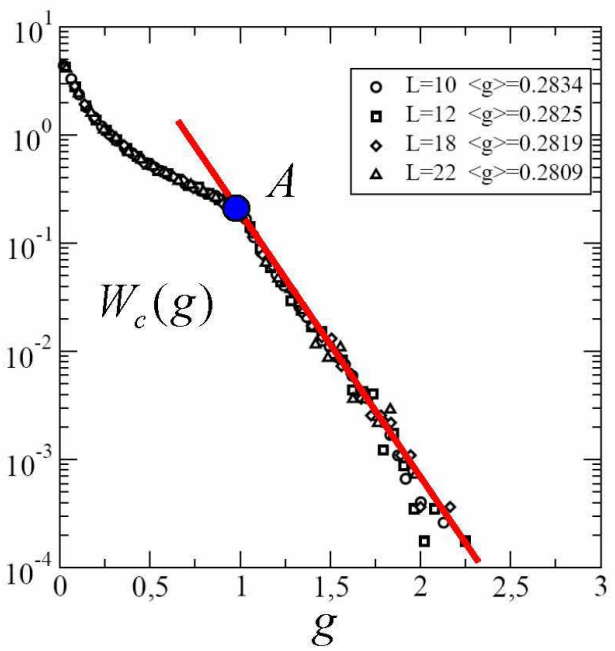

(a)

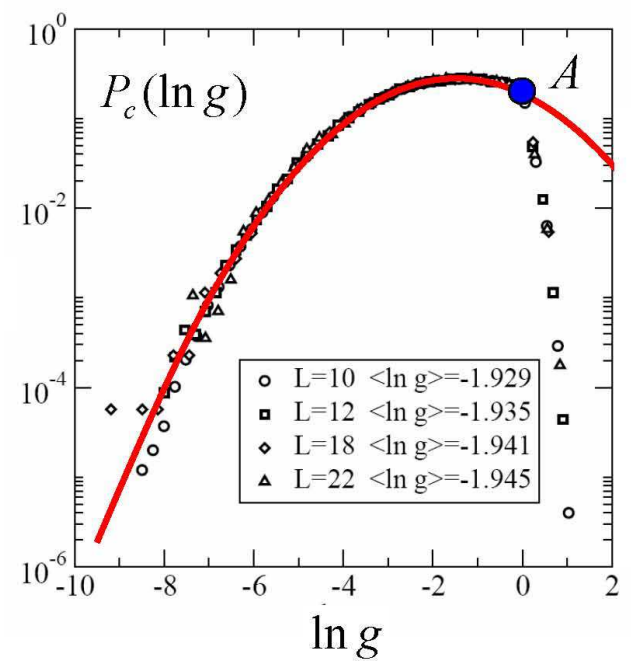

(b)

Figure 2: The distribution of conductance $W(g)$ (a) and of the conductance logarithm $P(\ln g)(W(g) d g \equiv$ $P(\ln g) d \ln g$ ) (b) according to numerical data by Markos [11. On the right of point $A$ the logarithm of $W(g)$ is linear in $g$, and on the left of $A$ the logarithm of $P(\ln g)$ is quadratic in $\ln g$. 
established in 21] using the self-consistent theory of localization [22; its validity for the second parameter also looks very probable.

\section{The Shapiro approach}

According to the Shapiro model [6, 7], the $d$ dimensional system is composed of one-dimensional chains, which are considered to be independent of each other, i.e. separated by dielectric interlayers (Fig.3,a). A distribution of resistances $P_{L}(\rho)$ for each chain is described by the equation

$$
\frac{\partial P_{L}(\rho)}{\partial L}=\alpha \frac{\partial}{\partial \rho}\left[\left(\rho^{2}+\rho\right) \frac{\partial P_{L}(\rho)}{\partial \rho}\right],
$$

which was derived by various authors [15- 18 and is considered to be sufficiently universal. The parameter $\alpha$ is proportional to the dispersion of a random potential and defined by relation $\langle\rho\rangle=\alpha L$ for small $L$; it has a sense of the inverse correlation length of the $1 D$ system. To obtain a description of the $d$-dimensional system composed of $n=b^{d-1}$ onedimensional chains, one should introduce the distribution $W(g)$, corresponding to $P(\rho)$,

$$
W(g)=\int P(\rho) \delta(g-1 / \rho) d \rho=g^{-2} P(1 / g)
$$

and find a distribution of the sum of $n=b^{d-1}$ random quantities with the same distribution $W(g)$. It can be made by introducing the characteristic function $F(t)=\left\langle e^{i g t}\right\rangle$ and raising it to the power $n$. The equation for $W(g)$, corresponding to (4), is obtained by substitutions $P=g^{2} W, \rho=1 / g$ :

$$
\frac{\partial W(g)}{\partial L}=\alpha\left[2 g(1+g) W(g)+g^{2}(1+g) W_{g}^{\prime}(g)\right]_{g}^{\prime} .
$$

Instead of the characteristic function, it will be more convenient to use the Laplace transform

$$
F(\tau)=\int_{0}^{\infty} e^{-\tau g} W(g) d g
$$

obtained by the change $i t \rightarrow-\tau$. Multiplying (6) by $e^{-\tau g}$ and integrating over $g$, one obtains the equation for $F(\tau)$ corresponding to the $1 D$ chain

$$
\begin{aligned}
F_{L+\Delta L}(\tau)= & F_{L}(\tau)+\alpha \Delta L\left[-\tau^{2} F_{L}^{\prime \prime \prime}(\tau)+\right. \\
& \left.+\tau(\tau-1) F_{L}^{\prime \prime}(\tau)\right]
\end{aligned}
$$

which we have written for finite increments. Raising $F_{L}(\tau)$ to the power $n=b^{d-1}$ and setting $b=$
$1+\Delta L / L$, we have an additional term $(\Delta L / L)(d-$ 1) $F_{L} \ln F_{L}$ in Eq.8, and finally 7

$$
\begin{aligned}
\frac{\partial F(\tau)}{\partial \ln L}=\alpha L[ & -\tau^{2} F^{\prime \prime \prime}(\tau)+\tau(\tau-1) F^{\prime \prime}(\tau)+ \\
& +p F(\tau) \ln F(\tau)]
\end{aligned}
$$

where $p=(d-1) / \alpha L$. The quantity $\alpha L$ has a sense of $L / \xi$, and evolution in $L$ for fixed $L / \xi$ leads to a stationary distribution, corresponding to the large length scales. Equation (9) describes the transient process, when $L$ is increasing from the atomic scale $a$ to scales of the order $\xi$. Eq.9 gives the adequate description for the model of Fig.3, a, but in fact was not investigated in Shapiro's papers 6, 7]. Instead, the simplified scheme was elaborated (Sec.5), where all $b^{d-1}$ chains were taken to be identical. This scheme did not allow to obtain the correct results for the metallic phase, and the whole approach was admitted unpromising by the author himself.

\section{Modification of the method}

In fact, after a certain modification the Shapiro scheme becomes very fruitful. First of all, let us change an interpretation of the model, coming from the artificial construction of Fig.3, a to a more adequate version presented in Fig.3,b. It is commonly accepted 23, that in the strong disorder regime conductance is determined by the resonant channels. There is a finite probability, that along a properly chosen trajectory the fluctuations of the random potential will be essentially less that in average over the system. An increased (in comparison with $L$ ) length of the trajectory is compensated by a more essential diminishing of $\alpha$, so conductance $g \sim \exp (-\alpha L)$ of the resonant channel will be exponentially greater than conductance of a typical chain in the Shapiro model (Fig.3,a)4. Thus, we naturally come to the notion of $1 D$ chains inserted

\footnotetext{
${ }^{4}$ For illustration, consider an example of the resonant trajectory on the $d$-dimensional cubical lattice. Let the trajectory starts on the left side of the system and is constructed by the following algorithm. If the trajectory comes to a certain point $A$, then it can be continued along $2 d$ directions to the nearest neighbors. We exclude of these continuations the directions to the left and backward along the trajectory; as for the rest of continuations, we choose the direction to the site with the minimal value of the random potential. It is easy to understand that (for large $d$ ) the length of the trajectory will be $\sim L d$, while the amplitude $W$ of the random potential along it will be approximately $d$ times less than in average. Since $\alpha \sim W^{2}$, then a value of $\alpha L$ will be approximately $d$ times smaller than for a typical straight-line trajectory.
} 


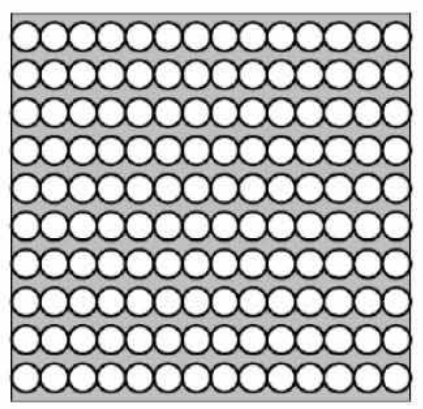

(a)

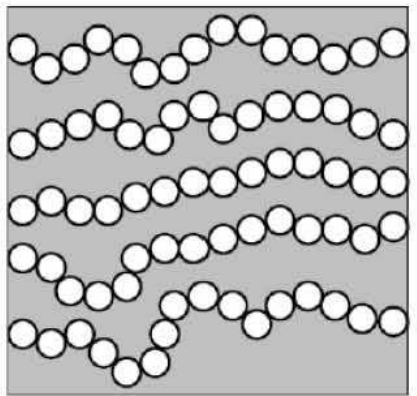

(b)

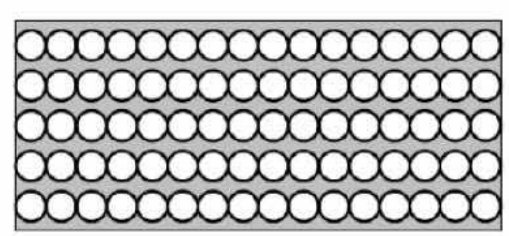

(c)

Figure 3: (a) In the Shapiro model, the $d$-dimensional system is composed of $b^{d-1}$ one-dimensional chains, consisting of $b$ atoms, inserted in the dielectric medium. (b) A more adequate interpretation corresponds to the change of the artificial construction of Fig.3, a by a notion of the resonant channels. (c) The "straighten" version of Fig.3,b.

in the dielectric medium. In this interpretation we easily remove the internal inconsistency of the initial model; indeed, a parallel connection of $L^{d-1}$ chains with conductance $\exp (-\alpha L)$ gives

$$
g \sim L^{d-1} \exp (-\alpha L)
$$

which tends to zero in the large $L$ limit. In fact, statistics of the resonant channels essentially depends on $L$, and the analogous dependence arises for the parameter $\alpha$, making the zero limit in (10) to be not obligatory. 5

Since the typical length of the resonant trajectories is somewhat greater than $L$, and their number is proportional to the cross-section area but somewhat less than $b^{d-1}$, so the "straighten" version of Fig.3,b corresponds to Fig.3,c, i.e. a cube is transformed into a parallelepiped. If the sides of the parallelepiped have the same scaling in $L$, then the system is topologically $d$-dimensional and its conductance distribution $W(g)$ possesses all qualitative properties of the $d$-dimensional distribution. Nevertheless, there is a quantitative difference related with transformation of the cube into the parallelepiped, which is an uncontrollable effect in the Shapiro scheme. The magnitude of this effect depends on the specific situation. Indeed, for weak disorder we practically

\footnotetext{
5 The $L$ dependence of $\alpha$ was introduced by Shapiro on the physical grounds, but there is no reasons for it in the model of Fig.3,a.
}

return to the initial model (Fig.3,a), since conductances of $1 D$ chains fluctuate weakly and the current streamlets are practically parallel to transversal boundaries of the system; so the dielectric interlayers have practically no effect.

Analogous conclusions can be drawn, if a situation is considered for the large length scales. In fact, the Shapiro approach implies the large-scale constructions: firstly, $b$ cubes are connected successively to form a quasi-one-dimensional system (Fig.4), and then a parallel connection of $b^{d-1}$ quasi-1D chains composes the $d$-dimensional system. For large $L$, a concentration of the auxiliary dielectric phase isolating quasi-1D chains becomes smaller and its influence reduces, suggesting validity of the Shapiro scheme in the large-scale limit. However, it was assumed implicitly that properties of quasi-1D systems are the same as those of strictly one-dimensional chains. This assumption is partially correct but needs an additional argumentation. It is commonly accepted that all properties of the cubic system of size $L$ are completely determined by the ratio $L / \xi$, so

$$
g=F(L / \xi)
$$

in accordance with the one-parameter scaling hypothesis 56. If cubes are connected to form a

\footnotetext{
${ }^{6}$ It would be more correctly to write that the distribution $W(g)$ is determined by the ratio $L / \xi$, but we prefer a simplified notation.
} 

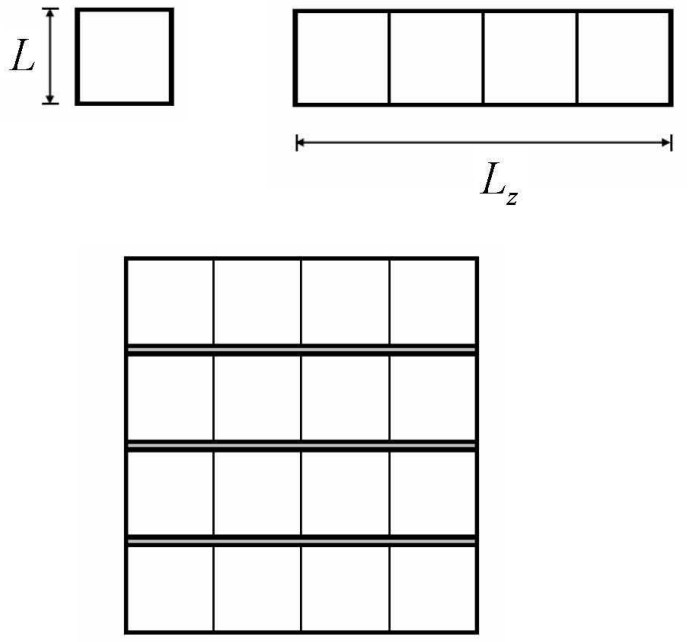

Figure 4: Large-scale constructions implied in the Shapiro scheme: the cubes of size $L$ are arranged into quasi-one-dimensional systems of length $L_{z}$, whose parallel connection composes the $d$-dimensional system.

quasi-1D system, then its conductance depends on the properties of the single block $(L / \xi)$ and a numbers of cubes $\left(b=L_{z} / L\right)$, i.e.

$$
g=F\left(L / \xi, L_{z} / L\right) .
$$

It is easy to verify that the distribution of $g$ depends essentially on each of two parameters, and not on a certain combination of them. Indeed, for the first two moments one has in the metallic phase

$$
\begin{aligned}
\langle g\rangle=\sigma L^{d-1} L_{z}^{-1} & =(L / \xi)^{d-2}\left(L / L_{z}\right), \\
\left\langle(\delta g)^{2}\right\rangle & =f\left(L_{z} / L\right),
\end{aligned}
$$

where the correlation length $\xi$ is introduced in the usual manner 24. The latter result follows from the theory of universal conductance fluctuations 1, 2]: since the constant $c$ in (1) depends on the space dimension, then the function $f(x)$ is equal $c_{d-1}$ for $x \ll 1$, comes through $c_{d}$ at $x=1$ and tends to $c_{1}$ for $x \gg 1$. Setting $L=a$ in Eq.12, we come to conclusion that a conductance distribution for a quasi1D system corresponds to a certain distribution of a strictly $1 \mathrm{D}$ system 7 . At this point we discover the

\footnotetext{
7 This statement is rigorous in the framework of orthodox scaling suggested in 5. In fact, the universal functions of type (12) arise only at large length scales, while at scales $\sim a$ they
}

real defect of the original Shapiro scheme: according to Eq.4, $P_{L}(\rho)$ is determined by one parameter $\alpha L$, and not two, as it follows from Eq.12.

In fact, equation (4) allows two-parameter generalization

$$
\frac{\partial P_{L}(\rho)}{\partial L}=\tilde{\alpha}\left[-\gamma(2 \rho+1) P_{L}(\rho)+\left(\rho^{2}+\rho\right) P_{L}^{\prime}(\rho)\right]^{\prime}
$$

(primes correspond to derivatives over $\rho$ ), which has practically the same level of universality. Indeed, description of $1 \mathrm{D}$ systems is conveniently made, considering each scatterer as a "black box", characterizing by a transfer-matrix $\hat{T}$, connecting amplitudes of plane waves on the left $\left(A e^{i k x}+B e^{-i k x}\right)$ and on the right $\left(C e^{i k x}+D e^{-i k x}\right)$ of the scatterer (Fig.5,a):

$$
\left(\begin{array}{c}
C \\
D
\end{array}\right)=\hat{T}\left(\begin{array}{c}
A \\
B
\end{array}\right) \text {. }
$$

If the scatterers are arranged successively (Fig.5,b), their transfer-matrices are multiplied. The matrix $\hat{T}$ is determined by the amplitudes of transmission $(t)$ and reflection $(r)$ and in the presence of time reversal invariance can be parametrized in the form 27 .

$$
\begin{gathered}
\hat{T}=\left(\begin{array}{cc}
1 / t^{*} & -r^{*} / t^{*} \\
-r / t & 1 / t
\end{array}\right)= \\
=\left(\begin{array}{cc}
\sqrt{\rho+1} e^{-i \varphi} & -\sqrt{\rho} e^{-i \theta} \\
-\sqrt{\rho} e^{i \theta} & \sqrt{\rho+1} e^{i \varphi}
\end{array}\right),
\end{gathered}
$$

where $\rho=|r / t|^{2}$ is the Landauer resistance [28]. For the product of large number $n$ of transfer-matrices, the distribution of $\varphi$ and $\theta$ is usually stabilized, i.e.

$$
P_{n}(\rho, \varphi, \theta)=P_{n}(\rho) P(\varphi, \theta) .
$$

If the phase distribution is uniform $(P(\varphi, \theta)=$ const), then we come to Eq.4, while in the general case we obtain Eq.14 with parameters (see Appendix 1)

$$
\gamma=\frac{1-2 A_{0}}{2 A_{0}}, \quad \tilde{\alpha}=2 \alpha A_{0}, \quad A_{0}=\left\langle\sin ^{2}(\varphi-\theta)\right\rangle .
$$

have a certain transient behavior. As was discussed by Wilson 19, such transient behavior can be excluded, if a model at small scales is chosen in the special manner (see the "ideal RG trajectory" in [25], which is approximately realized in the so called "improved" models [26]). Since for 1D systems we use the equation possessing the high level of universality (see below), the indicated "ideal" model will be also described by this equation. 


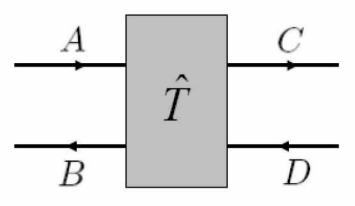

(a)

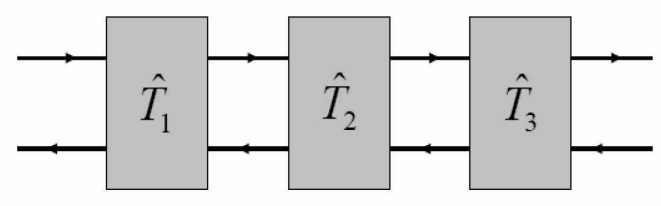

(b)

Figure 5: (a) For 1D systems, the transfer-matrix $\hat{T}$ relates amplitudes of plane waves on the left and on the right of a scatterer. (b) A successive arrangement of scatterers corresponds to multiplication of transfer-matrices.

It is easy to see inequality $\gamma \geq-1 / 2$, which will be essential for the phase diagram (Sec.6.5). For standard microscopical models, Eq.14 with variable $\gamma$ arises for small $L$, when the distribution of $\varphi$ and $\theta$ is not stabilized yet, and evolution of $\gamma$ is not universal. Equation (14) with constant $\gamma$ arises in lattice models due to effects of commensurability of the wave vector $k$ with a lattice spacing $a$ (when $k a$ is a rational number) 29]; it looks as a hardly observable exotic. However, there exist systematical reasons for appearing of equation (14), which are discussed in the next section 8 .

Equation (14) is of the diffusion type with a "time" $t=\tilde{\alpha} L$; so any initial distribution $P_{0}(\rho)$, localized in the region of small $\rho$, at large times transforms into the universal distribution, which has a different form in the region of small and large $\rho$ :

$$
\begin{array}{r}
P(\rho, t)=\frac{1}{\Gamma(\gamma+1)} \frac{\rho^{\gamma} \exp \{-\rho / t\}}{t^{\gamma+1}}, \quad \rho \lesssim 1 \\
P(\rho, t)=\frac{1}{\rho \sqrt{4 \pi t}} \exp \left\{-\frac{[\ln \rho-(2 \gamma+1) t]^{2}}{4 t}\right\},
\end{array}
$$

These results are obtained, if the main in $\rho$ terms are retained before $P_{L}(\rho)$ and $P_{L}^{\prime}(\rho)$ in Eq.14. The

\footnotetext{
${ }^{8}$ In a somewhat different context, a necessity of the twoparameter description of $1 \mathrm{D}$ systems was motivated in paper [30].
}

first distribution is close to Gaussian for large $\gamma$

$$
P(\rho, t)=\frac{1}{\sqrt{2 \pi \gamma t^{2}}} \exp \left\{-\frac{(\rho-\gamma t)^{2}}{2 \gamma t^{2}}\right\}
$$

and arises at large times when the diffusive spreading exceeds the width of the initial distribution $P_{0}(\rho)$. In the opposite case one can neglect the second term in the square brackets of (14) and obtain the automodel solution

$$
P(\rho, t)=e^{-2 \gamma t} P_{0}\left\{\left(\rho+\frac{1}{2}\right) e^{-2 \gamma t}-\frac{1}{2}\right\},
$$

reducing to a pure drift for $\gamma t \ll 1$

$$
P(\rho, t)=P_{0}(\rho-\gamma t) .
$$

If the initial distribution is Gaussian, then Eqs.21,23 correspond to the commonly accepted view on the conductance distribution in the metallic regime; this result was a stumbling block in the original version of the Shapiro method. Eq.20 corresponds to the lognormal distribution, which is commonly accepted for the localized regime.

\section{Semi-transparent boundaries}

Is was argued in 21], that for a correct definition of conductance of finite systems it is convenient to introduce semi-transparent boundaries separated the system from the ideal leads attached to it (Fig.6,a). The ideal leads are supposed to be sufficiently massive which is necessary for the correct interpretation of the linear response formulas. Indeed, the latter demand that the entering them $\delta$-functions were spread to the width $\Gamma$, which is tending to zero only after transition to the thermodynamic limit. In the case of finite systems, the thermodynamic limit is realized by increasing the size of ideal leads 31 . Such definition of conductance refers to the composite system "sample+ideal leads" and a question arises on its relation to the system under consideration.

If $k_{b}$ is a parameter, characterizing the effective transparency of interfaces, then the $k_{b}$ dependence of conductance has a view shown in Fig.6,b [21]: the linear behavior valid for small $k_{b}$ (when resistance is determined by weakly-transparent interfaces), is changed by saturation at $k_{b} \sim 1$, when the spreading of discrete levels of a finite system becomes comparable with their spacing, and a continuous density of states is formed. The physically reasonable definition of conductance corresponds to a value at the 


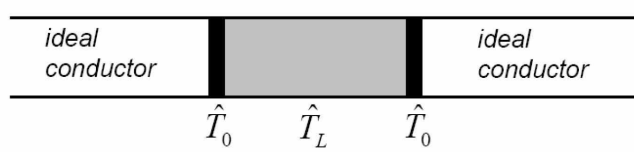

(a)

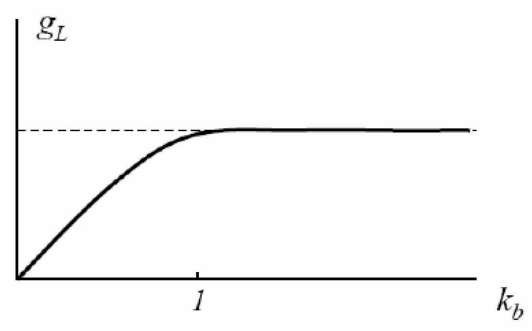

(b)

Figure 6: (a) For a correct definition of conductance of finite systems, it is useful to introduce semitransparent interfaces, separated the system from the ideal leads connected to it. (b) Conductance of a finite system as a function of the parameter $k_{b}$, having a sense of the effective transparency of the boundary.

plateau for $k_{b} \gtrsim 1$; however, one should not take too large $k_{b}$, because the "plateau" may correspond to a slow dependence arisen due to influence on the system of its environment. Instead of setting $k_{b} \sim 1$ in the dependence $g=f\left(k_{b}\right)$ one can take a derivative $d g / d k_{b}$ for $k_{b} \rightarrow 0$; 9 then conductance of the open system is defined in terms of almost closed systems 21. Such definition (a) surely refers to the system under consideration and does not depend on the properties of the environment, (b) is free from ambiguities related with exclusion of the contact resistance of the reservoir [32, and (c) provides infinite conductance for an ideal system [21]. It is natural to expect that introducing of semi-transparent boundaries will be also useful in discussion of conductance distributions; but it immediately leads to equation (14) with finite $\gamma$.

If $\hat{T}_{L}$ is the transfer-matrix for a system of size $L$, then a situation corresponding to Fig.6,a is described by the matrix

$$
\hat{T}_{L}^{\prime}=\hat{T}_{0} \hat{T}_{L} \hat{T}_{0}
$$

where $\hat{T}_{0}$ corresponds to the interface. The increase

\footnotetext{
9 These two procedures coincide only in the order of magnitude, but it should not arouse anxiety: only a ratio of conductances has a physical sense and variation of the definition by a constant factor corresponds to a change of the unit of measurement.
}

of the system length by $\Delta L$ gives

$$
\hat{T}_{L+\Delta L}^{\prime}=\hat{T}_{0} \hat{T}_{L} \hat{T}_{\Delta L} \hat{T}_{0}=\hat{T}_{L}^{\prime} \cdot \hat{T}_{0}^{-1} \hat{T}_{\Delta L} \hat{T}_{0},
$$

and corresponds to multiplication by the matrix $\hat{T}_{0}^{-1} \hat{T}_{\Delta L} \hat{T}_{0}$ close to the unit one, so the evolution equation can be derived by a standard method (see Appendix 1). If $\hat{T}_{L}$ is accepted in the form (16) and $\hat{T}_{0}$ corresponds to the point scatterer

$$
\hat{T}_{0}=\left(\begin{array}{cc}
1-i \kappa & -i \kappa \\
i \kappa & 1+i \kappa
\end{array}\right)
$$

then for $\kappa \gg 1$ one has a relation between parameters of $\hat{T}_{L}^{\prime}$ and $\hat{T}_{L}$ :

$$
\begin{gathered}
\rho^{\prime}=4 \kappa^{4}(\sqrt{\rho+1} \sin \varphi+\sqrt{\rho} \sin \theta)^{2}, \\
\operatorname{tg} \varphi^{\prime}=2 \kappa \frac{\sqrt{\rho+1}(\cos \varphi-\kappa \sin \varphi)-\kappa \sqrt{\rho} \sin \theta}{\sqrt{\rho+1}(\cos \varphi-2 \kappa \sin \varphi)-2 \kappa \sqrt{\rho} \sin \theta} \\
\operatorname{tg} \theta^{\prime}=-2 \kappa \frac{\sqrt{\rho+1}(\cos \varphi-\kappa \sin \varphi)-\kappa \sqrt{\rho} \sin \theta}{\sqrt{\rho} \cos \varphi} .
\end{gathered}
$$

It is easy to see that the distribution of $\varphi^{\prime}$ and $\theta^{\prime}$ is not trivial even for the uniform phase distribution $P(\varphi, \theta)$ of the initial matrix $\hat{T}_{L}$. In particular, for $\kappa \gg 1$ distributions of $\varphi^{\prime}$ and $\theta^{\prime}$ have a form of the narrow Lorentz peaks

$$
\begin{gathered}
P\left(\varphi^{\prime}\right)=\frac{1}{2 \pi} \frac{1 / 2 \kappa^{2}}{1 / 4 \kappa^{4}+\left(\delta \varphi^{\prime}+1 / \kappa\right)^{2}}, \\
P\left(\theta^{\prime}\right)=\frac{1}{2 \pi} \frac{1 / 2 \kappa^{2}}{1 / 4 \kappa^{4}+\left(\delta \theta^{\prime}\right)^{2}},
\end{gathered}
$$

where $\delta \varphi^{\prime}=\varphi^{\prime} \pm \pi / 2$ and $\delta \theta^{\prime}=\theta^{\prime} \pm \pi / 2$. We have taken into account that for $\rho \gg 1$ quantities $\varphi^{\prime}$ and $\theta^{\prime}$ does not depend on $\rho$; a certain dependence on $\rho$ is present for $\rho \lesssim 1$, but localization at $\delta \varphi^{\prime} \approx 1 / \kappa$ and $\delta \theta^{\prime} \approx 0$ is retained as one can immediately see from (28), (29). Calculating the average value in (18) using distributions (30), (31), one has

$$
A_{0}=1 / \kappa^{2}, \quad \gamma=\kappa^{2} / 2 .
$$

We come to the following conclusion: if the phase distribution $P(\varphi, \theta)$ for the initial system is uniform, then introducing of weakly-transparent interfaces makes it strongly localized and leads to the evolution equation with $\gamma \gg 1$.

\section{Simplified schemes}


In the complete version of the Shapiro approach, an evolution of the distribution is described by a nonlinear equation for $F(\tau)$, which is somewhat cumbersome for investigation. There is a certain methodical interest in formulation of approximate schemes leading to the more simple equations.

\subsection{Shapiro's scheme for average quantities}

Multiplying (4) by $\rho$ and integrating, one has the closed equation for the average resistance of the $1 \mathrm{D}$ system, whose solution

$$
\bar{\rho}_{L}=\frac{1}{2}\left(e^{2 \alpha L}-1\right)
$$

coincides with known results [7, 27, 28]. Composing a system of length $b L$ by successive connection of $b$ blocks of size $L$, one has a scale transformation for the $1 D$ chain

$$
\bar{\rho}_{b L}^{(1)}=\frac{1}{2}\left[\left(1+2 \bar{\rho}_{L}^{(1)}\right)^{b}-1\right],
$$

while the parallel connection of $b^{d-1}$ chains composes the $d$-dimensional system:

$$
\bar{\rho}_{b L}=\frac{1}{2} b^{-(d-1)}\left[\left(1+2 \bar{\rho}_{L}\right)^{b}-1\right] .
$$

Taking $b$ close to unity, one has the differential equation, which can be rewritten in terms of the variable $g_{L}=1 / 2 \bar{\rho}_{L}[7]$

$$
\frac{d \ln g_{L}}{d \ln L}=d-1-\left(1+g_{L}\right) \ln \left(1+1 / g_{L}\right) \equiv \beta\left(g_{L}\right)
$$

and has a form expected from one-parameter scaling [5]. Equation (36) gives a qualitative description of the Anderson transition and reproduces the correct result $\nu=1 / \epsilon$ for the critical exponent $\nu$ of the correlation length in the space of dimension $d=2+\epsilon$. The latter is not surprising, since the only essential assumption is made in proceeding from (34) to (35): for the parallel connection of chains one should sum average conductances, i.e. $\bar{g}_{b L}=b^{d-1} \bar{g}_{b L}^{(1)}$ instead of the exploited relation $\bar{\rho}_{b L}=b^{-(d-1)} \bar{\rho}_{b L}^{(1)}$. The latter is valid approximately for a narrow distribution, which is the case for $d=2+\epsilon$.

Using equation (14) instead of (4) and taking into account that $\tilde{\alpha}(\gamma+1)=\alpha$ according to (18), one can see that dependence on $\gamma$ disappears and the result (33) retains for $\bar{\rho}_{L}$; so Eqs.34-36 remain unchanged. Therefore, the modified Shapiro scheme leads to the correct critical behavior of $\xi$, so far as this behavior can be controlled.

\subsection{Shapiro's scheme for $P(\rho)$}

In a simplified variant of the Shapiro approach, all chains in Fig.3, a are assumed to be identical, so $P_{L}(\rho)=b^{d-1} P_{L}^{(1)}\left(b^{d-1} \rho\right)$ and instead of (9) one has a linear equation for $P_{L}(\rho)$

$$
\begin{aligned}
& \frac{\partial P_{L}(\rho)}{\partial \ln L}=A\left[-\gamma(2 \rho+1) P_{L}(\rho)+\right. \\
& \left.\quad+\rho(\rho+1) P_{L}^{\prime}(\rho)+p \rho P_{L}(\rho)\right]^{\prime}
\end{aligned}
$$

where $A=\tilde{\alpha} L$. It has the stationary solution

$$
P_{c}(\rho)=\text { const } \frac{\rho^{\gamma}}{(\rho+1)^{p-\gamma}},
$$

coinciding with (3) for $\gamma=0$. Accepting the result $\bar{\rho}=\epsilon$ for $d=2+\epsilon$, following from (36), one has $p=(\gamma+1) / \epsilon$. In opposite to Shapiro's result $(3)$, the distribution (38) provides finite values of moments $\left\langle g^{n}\right\rangle$ for $n \lesssim \gamma$. Setting $\gamma \sim 1 / \epsilon^{2}$ and calculating the Laplace transform (7) by the saddle-point method, one has

$$
F(\tau)=\exp \left\{\frac{1}{\epsilon^{2}} f(\epsilon \tau)\right\},
$$

where $f(x)$ has a regular expansion and provides correct results for the cumulants determined by the first relation (2); the second relation (2) is not reproduced in this scheme.

\subsection{A simplified scheme for $d=2+\epsilon$}

A more adequate approximation can be formulated having in mind a situation for $d=2+\epsilon$. According to (2), the cumulants $\left\langle\left\langle g^{n}\right\rangle\right\rangle$ decrease fast with $n$, so in the main order in $\epsilon$ one can set $\ln F(\tau) \approx$ const $\tau$. Not difficult to trace that it is equivalent to appearing the term $\rho^{2} P$ in the square bracket of equation for $P(\rho)$

$$
\begin{aligned}
\frac{\partial P(\rho)}{\partial \ln L}=A & {\left[c \rho^{2} P(\rho)-\gamma(2 \rho+1) P(\rho)+\right.} \\
& \left.+\rho(\rho+1) P^{\prime}(\rho)\right]^{\prime} .
\end{aligned}
$$

Till the present moment we used the Landauer definition of resistance $\rho=|r / t|^{2}$ [28. The alternative is given by the Economou-Soukoulis definition $\tilde{\rho}=|1 / t|^{2}[31$, so $\rho=\tilde{\rho}-1$. Taking into account ambiguities, related with exclusion of the contact resistance of the reservoir [32, and a change of the $\rho$ normalization in the course of transition to quasi-1D systems, we should generally make a 
change $\rho \rightarrow \rho-\rho_{0}$, where $\rho_{0}$ depends on details of the definition. However, in the case of weaklytransparent boundaries, a scale of $\rho$ is increased by a factor $\kappa^{4}$ (see $(27)$ ), so the terms containing $\rho_{0}$ are insignificant and can be omitted. Such universal equation (where $\rho(\rho+1)$ is replaced by $\rho^{2}$ ), obtained in the limit $\kappa \rightarrow \infty$, can be extrapolated into the region $\kappa \sim 1$ : it exactly corresponds to a procedure suggested in [21], when the dependence $g=$ const $k_{b}$, obtained in the limit $k_{b} \rightarrow 0$, is extrapolated to value $k_{b}=1$. Replacement $\rho \rightarrow \rho-\rho_{0}$ should be made also in other terms of (40), where a situation is more complicated due to unknown behavior of parameters in the course of the described procedure; in fact, the effect of the change $\rho \rightarrow \rho-\rho_{0}$ can be removed by redefinition of parameters $c, \gamma$ and the change of the general $\rho$ scale. As a result, we have the equation for $P(\rho)$

$$
\begin{gathered}
\frac{\partial P(\rho)}{\partial t}=\left[\left(c \rho^{2}-2 \gamma \rho-\gamma\right) P(\rho)+\rho^{2} P^{\prime}(\rho)\right]^{\prime} \\
t=A \ln L
\end{gathered}
$$

which gives the equation for $W(g)$ of the same structure

$\frac{\partial W(g)}{\partial t}=\left[\left(\gamma g^{2}+2(\gamma+1) g-c\right) W(g)+g^{2} W^{\prime}(g)\right]^{\prime}$,

and explains the strange analogy between $P(\rho)$ and $W(g)$, discovered by Shapiro. The stationary solution has a form

$$
W_{c}(g)=\text { const } g^{-2(\gamma+1)} \exp \{-c / g-\gamma g\}
$$

and provides a finiteness of all moments of conductance. Calculating the Laplace transform by the saddle-point method and setting $\gamma \sim 1 / \epsilon, c \sim 1 / \epsilon^{3}$, we obtained the result of type (39), providing validity of the first relation (2).

For evolution of moments one has from (42)

$$
\frac{\partial\left\langle g^{n}\right\rangle}{\partial t}=c n\left\langle g^{n-1}\right\rangle+n(n-2 \gamma-1)\left\langle g^{n}\right\rangle-\gamma n\left\langle g^{n+1}\right\rangle
$$

and deviations $x_{n}=\left\langle g^{n}\right\rangle-\left\langle g^{n}\right\rangle_{c}$ from the stationary values obey the same equation. If the latter are proportional to $\exp (\lambda t)$, then we have a three-diagonal matrix for determination of the $\lambda$ spectrum. The eigenvalue $\lambda_{n}$ is determined by the matrix of size $n \times n$ and corresponds to nonzero deviations $x_{1}$, $x_{2}, \ldots, x_{n}$, obtained for the boundary conditions $x_{0}=x_{n+1}=0$. The indicated matrix is not Hermitian and its eigenvalues are complex; one can find by the quasi-classical method, that

$$
\operatorname{Re} \lambda_{n}=\frac{n^{2} / 2-(2 \gamma+1) n}{\ln n}, \quad n \lesssim 1 / \epsilon^{2} .
$$

In the region of values $n \sim 1 / \epsilon$ the denominator can be replaced by $\ln (1 / \epsilon)$, and one obtains for evolution of moments

$$
\left\langle g^{n}\right\rangle \sim e^{\lambda_{n} t} \sim L^{\tilde{A}\left[n^{2}-2(2 \gamma+1) n\right]},
$$

where $\tilde{A}=A / 2 \ln (1 / \epsilon)$. To reproduce the second relation in (2) one should accept $\tilde{A}=\epsilon,(2 \gamma+1) \tilde{A}=1$, so $\gamma \sim 1 / \epsilon$ in agreement with the condition for validity of (39). In the described simplified scheme, the second result (2) is reproduced with the logarithmic accuracy; in the complete theory (see below) it will be obtained precisely.

\section{Conductance distribution in the complete theory}

\subsection{The main equations}

It is clear from above considerations, that for $1 \mathrm{D}$ systems we should accept equation (14) with replacement $\rho \rightarrow \rho-\rho_{0}$. Then we should take a limit of weakly-transparent interfaces to obtain the universal equation, and extrapolate it to transparency of the order of unity; practically it reduces to replacements $\rho(\rho+1) \rightarrow \rho^{2}$ and $\gamma\left(1-2 \rho_{0}\right) \rightarrow \tau_{0}$. As a result, the equation for $F(\tau)$ in the $d$-dimensional case has a form

$$
\begin{gathered}
\frac{\partial F(\tau)}{\partial t}=\tau\left(\tau+\tau_{0}\right) F^{\prime \prime}(\tau)-2 \gamma \tau F^{\prime}(\tau)+p F(\tau) \ln F(\tau) \\
t=A \ln L
\end{gathered}
$$

where the parameter $\tau_{0}$ should be positive to avoid singularities on the positive semi-axis; it specifies the general scale of conductance, which is not controlled in the theory. Introducing a variable $u$ by relation $F(\tau)=\exp \{u(\tau)\}$, we have

$$
\frac{\partial u}{\partial t}=\tau\left(\tau+\tau_{0}\right)\left[u^{\prime \prime}+u^{\prime 2}\right]-2 \gamma \tau u^{\prime}+p u .
$$

The stationary version of equation (48) is of the main interest, since this equation describes the transient behavior to the limit of large length scales (see Footnote 7 ) for fixed values of $L / \xi$ and $L_{z} / L$. As a result, 
$\xi$ is increasing to infinity, and all obtained distributions correspond to the Anderson transition point, differing in values of two indicated parameters 10. A stationary configuration can be sought in the form of the regular expansion

$$
u(\tau)=\sum_{n=1}^{\infty} B_{n} \tau^{n}
$$

where a zero-order term is absent due to normalization of $W(g)$ (see $(7))$. Substituting to (48), one has for the expansion coefficients

$$
\begin{gathered}
(p-2 \gamma) B_{1}+\tau_{0} B_{1}^{2}+2 \tau_{0} B_{2}=0 \\
(p-4 \gamma) B_{2}+4 \tau_{0} B_{1} B_{2}+6 \tau_{0} B_{3}+2 B_{2}+B_{1}^{2}=0 \\
(p-6 \gamma) B_{3}+4 \tau_{0} B_{2}^{2}+6 \tau_{0} B_{1} B_{3}+12 \tau_{0} B_{4}+ \\
+6 B_{3}+4 B_{1} B_{2}=0
\end{gathered}
$$

and so on.

\subsection{Reproducing the results for $d=2+\epsilon$}

Coefficients $B_{n}$ are proportional to the cumulants $\left\langle\left\langle g^{n}\right\rangle\right\rangle$ and for $d=2+\epsilon$ they obey the hierarchy $B_{1} \gg B_{2} \gg B_{3} \gg \ldots$ (see $(2)$ ); so we can omit the term with $B_{2}$ in the first equation, the term with $B_{3}$ in the second equation, etc., which corresponds to neglecting the term $\tau \tau_{0} u^{\prime \prime}$ in Eq.48. After it, coefficients $B_{1}, B_{2}, \ldots$ are determined uniquely and for $p \gg 1, \gamma \ll p$ are estimated as $B_{n} \sim p / \tau_{0}^{n}$. It suggests substitution $u(\tau)=p f\left(\tau / \tau_{0}\right)$ and the main order in $p$ gives the equation for $f(x)$

$$
x(x+1) f^{\prime 2}+f=0 .
$$

Its solution leads to the result

$$
F(\tau)=\exp \left(-p \operatorname{Arsh}^{2} \sqrt{\tau / \tau_{0}}\right)
$$

and the necessary form (39) is obtained for $\tau_{0} \sim 1 / \epsilon$, $p \sim 1 / \epsilon^{2}$. Producing the inverse Laplace transformation

$$
W(g)=\frac{\tau_{0}}{2 \pi i} \int_{-i \infty}^{i \infty} d x \exp \left\{-p \operatorname{Arsh}^{2} \sqrt{x}+\tau_{0} g x\right\},
$$

\footnotetext{
10 "The critical distribution", discussed in [6, 7, 8, and other papers, corresponds to a situation $L_{z}=L, L / \xi=0$. These two conditions determine the critical values $p_{c}$ and $\gamma_{c}$ for two parameters entering (48). We do not try to calculate these parameters for any specific situations but investigate all family of distributions in whole. In the framework of one-parameter scaling, the values $p_{c}$ and $\gamma_{c}$ should depend only on $d$ and the boundary conditions.
}

and calculating the integral in the saddle-point approximation, we have after setting $g_{c}=p / \tau_{0}$

$$
\begin{gathered}
W(g) \sim \frac{1}{g} \exp \left\{-\frac{p}{4}\left(\ln \frac{a(g) g_{c}}{g}\right)^{2}\right\}, \quad g \ll g_{c} \\
W(g) \sim \exp \left\{-\frac{3}{4} p\left(\frac{g-g_{c}}{g_{c}}\right)^{2}\right\}, \quad\left|g-g_{c}\right| \ll g_{c} \\
W(g) \sim\left(\frac{g_{c}}{g}\right)^{3 / 2} \exp \left\{-\tau_{0} g\right\}, \quad g \gg g_{c} \quad(54 c)
\end{gathered}
$$

where $a(g)$ is logarithmically varying function. Eqs.54a and $54 b$ can be formally united, if $a(g)$ is accepted to tend to unity for $g \rightarrow g_{c}$. For $p \sim 1$, a difference of $a(g)$ from a constant is practically inessential, and the log-normal asymptotics (54a), obtained formally for small $g$, describes satisfactorily a vicinity of the maximum $g_{c}$; together with (54c) it explains the situation demonstrated in Fig.2.

Consider evolution of cumulants, if the distribution is deviated from the stationary one. Assuming the deviations to be proportional to $\exp (\lambda t)$, we have the equation

$$
\tau\left(\tau+\tau_{0}\right) u^{\prime 2}+p u=-\tau\left(\tau+\tau_{0}\right) u^{\prime \prime}+2 \gamma \tau u^{\prime}+\lambda u,
$$

whose solution is known in the absence of the righthand-side. The latter can be taken into account iteratively, and setting $u=u_{c}+\delta u$ one has

$$
\hat{L} \delta u=f\{u\}
$$

where

$$
\begin{gathered}
\hat{L} \delta u \equiv \tau\left(\tau+\tau_{0}\right) 2 u_{c}^{\prime} \delta u^{\prime}+p \delta u, \\
f\{u\} \equiv-\tau^{2} u^{\prime \prime}+2 \gamma \tau u^{\prime}+\lambda u,
\end{gathered}
$$

and the term $\tau \tau_{0} u^{\prime \prime}$ is omitted, since it is inessential for the actual solution (see beginning of Sec.6.2). The operator $\hat{L}$ is proportional to $p$, and substitution $u=u_{c}$ into the right-hand-side of (56) gives a correction $\delta u \sim u_{c} / p$ to the previously found solution. Now, if we introduce a perturbation of the form

$$
u=u_{n}(\tau)=B_{n} \tau^{n}, \quad \lambda=\lambda_{n}=n^{2}-(2 \gamma+1) n
$$

in the right-hand-side of (56), then it is easy to see that $f\{u\}=0$ and $\delta u=0$. Thus, in a framework of the iterative procedure the perturbation (58) does not violate the validity of Eq. 55 and can have a nonstationary evolution. Since coefficients $B_{n}$ are proportional to cumulants $\left\langle\left\langle g^{n}\right\rangle\right\rangle$, then

$$
\left\langle\left\langle g^{n}\right\rangle\right\rangle \sim e^{\lambda_{n} t} \sim L^{A\left[n^{2}-(2 \gamma+1) n\right]}
$$



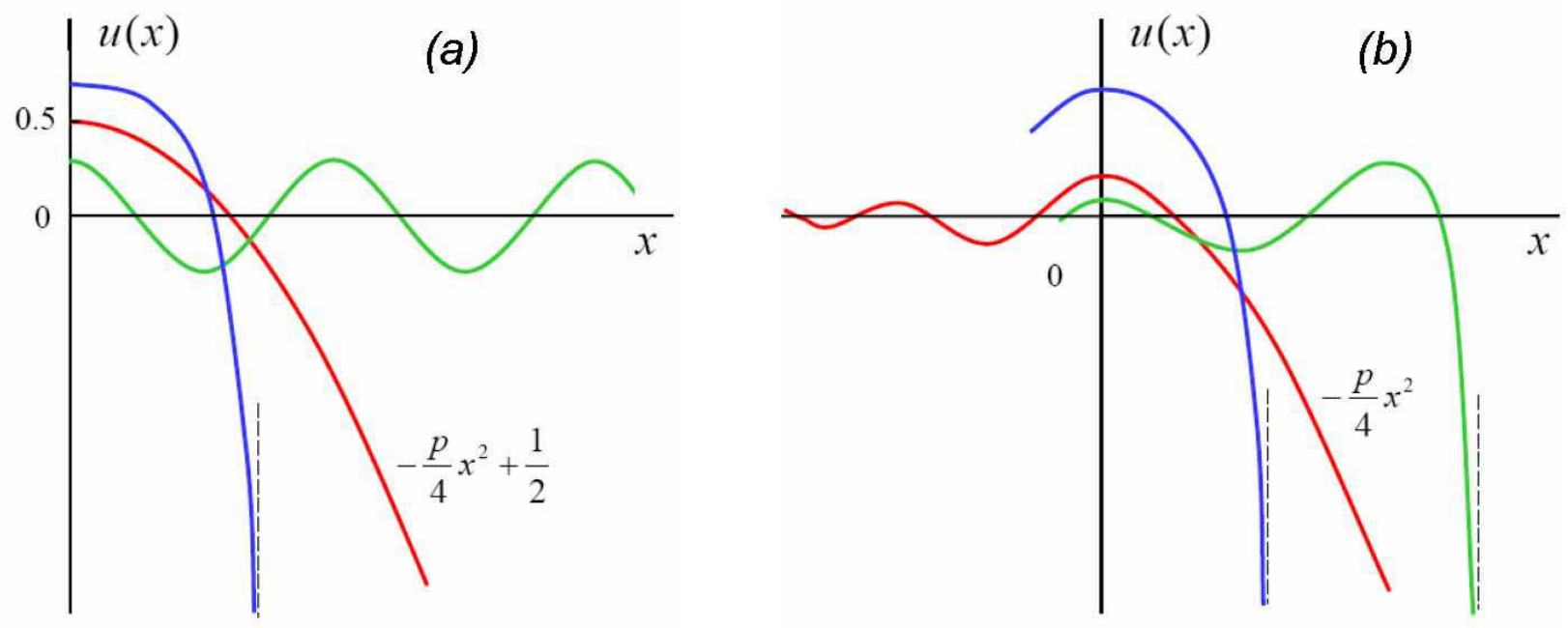

Figure 7: (a) Investigation of the quadrature (61) of Eq.60 with $\tilde{\gamma}=0$ leads to existence of three types of solutions: quadratic for $u(0)=1 / 2$, periodic for $u(0)<1 / 2$ and logarithmically divergent at a finite point for $u(0)>1 / 2$. (b) In the case $\tilde{\gamma}>0$, only the solution with a quadratic asymptotics remains regular; all other solutions, increasing from the region of negative $x$, break to a logarithmic singularity, when their amplitude of oscillations becomes of the order of unity.

and with a choice $A=\epsilon,(2 \gamma+1) A=2$ we reproduce the second relation (2).

It should be noted that our analysis is not restricted by reproducing (2), but gives the closed expression (53) for the critical distribution in the space dimension $d=2+\epsilon$.

\subsection{Uniqueness of the physical solution}

We have seen above that in the main order in $p$ coefficients $B_{n}$ are determined unambiguously, providing uniqueness of the stationary solution $u_{c}(\tau)$. If the omitted terms are taken into account iteratively, and equation (56) is solved by variation of constants, then $\delta u(\tau)=f_{\text {reg }}(\tau)+C \operatorname{Arsh} \sqrt{\tau / \tau_{0}}$, where $f_{\text {reg }}(\tau)$ is a regular function. The correction to $u_{c}(\tau)$ is determined uniquely, if $u(\tau)$ is accepted to be regular at zero. As we see below, such situation retains in the general case.

For large $\tau$, we can replace $\tau\left(\tau+\tau_{0}\right)$ by $\tau^{2}$ in equation (48), and substitution $x=\ln \tau$ reduces its stationary version to the form

$$
u_{x x}^{\prime \prime}+\left(u_{x}^{\prime}\right)^{2}-\tilde{\gamma} u_{x}^{\prime}+p u=0,
$$

where $\tilde{\gamma}=2 \gamma+1$. For $\tilde{\gamma}=0$, Eq.60 is integrated in quadratures 33 .

$$
x=C_{1}+\int \frac{d u}{\sqrt{Y}}, \quad Y=C_{2} e^{-2 u}+\frac{p}{2}(1-2 u),
$$

and investigation of (61) leads to the picture presented in Fig.7,a. Invariance relative to changes $x \rightarrow-x$ and $x \rightarrow x+x_{0}$ allows to consider only solutions even in $x$, whose derivative at $x=0$ is zero. If $u(0)=1 / 2$, then Eq.60 has a simple solution

$$
u(x)=-\frac{p}{4} x^{2}+\frac{1}{2} ;
$$

if $u(0)<1 / 2$, then solutions are periodical; if $u(0)>1 / 2$, then solutions diverge logarithmically at a finite point $x_{c}$ (see Fig.7,a). In fact, all characteristic regimes are determined by domination of two (from three) terms in Eq.60 with $\tilde{\gamma}=0$ : domination of $u^{\prime 2}$ and $p u$ leads to the quadratic solution $u \sim x^{2}$, domination of $u^{\prime \prime}$ and $p u$ provides the periodic solution $u \sim \cos \sqrt{p} x$, domination of $u^{\prime \prime}$ and $u^{\prime 2}$ results in the logarithmic singularity $u \sim \ln \left(x-x_{c}\right)$.

In the case $\tilde{\gamma}>0$, invariance respective $x \rightarrow-x$ is violated and the periodic solutions acquire a negative damping decrement. As a result, the quadratic solution (transforming to oscillations decreasing in the negative $x$ direction) remains the only regular 
one. All other solutions break to the logarithmic singularity after reaching the amplitude of oscillations of the order of unity (Fig.7,b).

According to the definition (7), the function $F(\tau)$ is regular and monotonically decreasing; so in the region $x=\ln \tau \gg 1$ only the quadratic solution is physically satisfactory; it has one-parameter indeterminacy related with shifts along the $x$ axis. In the region $\tau \lesssim 1$ one should return to the initial equation (48), and the one-parameter freedom is removed by condition $u(0)=0$, following from normalization of $W(g)$.

\subsection{Universal tails}

A typical behavior of the function $u(\tau)$ is represented in Fig.8. For large $\tau$ it has the asymptotics

$$
u(x)=-\frac{1}{4} p\left(\ln \tau-x_{0}\right)^{2},
$$

which in the saddle-point approximation gives the log-normal tail (54a) in the small $g$ region.

The asymptotics of a distribution for large $g$ is determined by a singularity at the point $\tau=-\tau_{0}$, in whose vicinity the general solution has a form

$$
u(\tau)=C_{1}\left(\tau+\tau_{0}\right)^{1+2 \gamma}+C_{2} .
$$

Substitution of (64) into the inverse Laplace transform gives the exponential behavior for large $g$ :

$$
W(g) \sim g^{-2-2 \gamma} \exp \left(-\tau_{0} g\right), \quad g \rightarrow \infty .
$$

This result is more correct than $(54 \mathrm{c})$, where the character of singularity at $\tau=-\tau_{0}$ was somewhat distorted due to the use of approximation (52).

As a result, the tails of the distribution $W(g)$ are universal, but their physical actuality depends on the specific situation (see below).

\subsection{Phase diagram 11}

Calculating corrections to (52), including the omitted terms iteratively, one has for the coefficients $B_{1}$ and $B_{2}$

$$
-B_{1}=p-2 \gamma-\frac{2}{3}, \quad 3 B_{2}=p-\frac{8}{3} \gamma-\frac{38}{45} .
$$

Since $B_{1}=-\langle g\rangle, 2 B_{2}=\left\langle(\delta g)^{2}\right\rangle$, then constant values of $\langle g\rangle$ and $\left\langle(\delta g)^{2}\right\rangle$ correspond to straight lines in the $(p, \gamma)$ plane. Formally, equation (66) is valid for $p \gg 1, \gamma \ll p$, but practically it describes almost all phase diagram (Fig.9,a).

\footnotetext{
${ }^{11}$ In this section we set $\tau_{0}=1$. Results for arbitrary $\tau_{0}$ can be obtained by the change $\tau \rightarrow \tau / \tau_{0}$ or $g \rightarrow g \tau_{0}$ in final expressions.
}

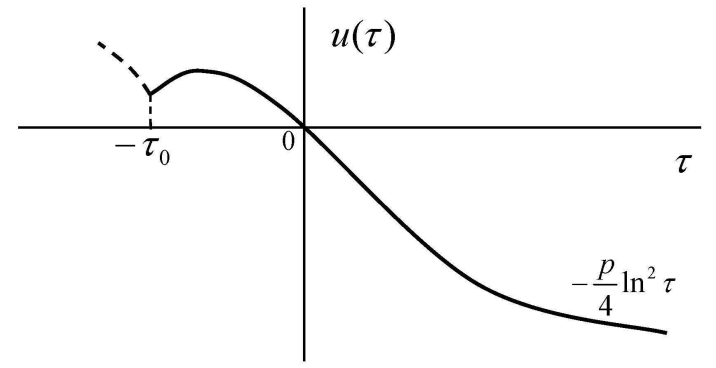

Figure 8: Behavior of the physical solution $u(\tau)$ on the real axis; for $\tau<-\tau_{0}$ it becomes complex.

The lower boundary of the physical region is determined by two dependencies $p=2 \gamma$ and $p=(1+$ $2 \gamma)^{2} / 4$, conjugated at the point $\gamma=0.5$ (Fig.9,b). In approaching the lower boundary, coefficients $B_{1}$ and $B_{2}$ tend to zero, maintaining condition $B_{2} \sim B_{1}$ for $\gamma<0.5, B_{2} \sim B_{1}^{2 \gamma}$ for $0.5<\gamma<1$ and $B_{2} \sim B_{1}^{2}$ for $\gamma>1$.

Indeed, due to positiveness of $B_{2}$ and negativeness of $B_{1}$ the first relation (50) leads to the inequality

$$
p-2 \gamma=\left|B_{1}\right|+2 B_{2} /\left|B_{1}\right| \geq 0,
$$

which gives a necessary condition $p \geq 2 \gamma$ for the physical solution. Setting $p=2 \gamma+\epsilon$, we can rewrite (48) in the form

$\hat{L} u \equiv \tau(\tau+1) u^{\prime \prime}-2 \gamma \tau u^{\prime}+2 \gamma u=-\tau(\tau+1) u^{\prime 2}-\epsilon u$.

Equation $\hat{L} u=0$ has a solution $u_{0}(\tau)=A \tau$, which allows to take the right-hand-side into account iteratively

$$
\hat{L} \delta u=-A^{2}\left(\tau^{2}+\tau\right)-\epsilon A \tau .
$$

For solvability of equation (68) its right-hand-side should be orthogonal to solution $v_{0}(\tau)$ of the conjugated equation $\hat{L}^{+} v_{0}=0$

$$
0=\left(v_{0}, \hat{L} \delta u\right)=-A^{2}\left(v_{0}, \tau^{2}+\tau\right)-\epsilon A\left(v_{0}, \tau\right),
$$

where $v_{0}(\tau)=(1+\tau)^{-1-2 \gamma}$. For $\gamma>1$, the integrals corresponding to scalar products $\left(v_{0}, \tau^{2}\right)$ and $\left(v_{0}, \tau\right)$ converge, so $A \sim \epsilon, \delta u \sim \epsilon^{2}$. Since the linear and quadratic in $\tau$ terms contain in $u_{0}(\tau)$ and $\delta u(\tau)$ correspondingly, then $B_{1} \sim \epsilon, B_{2} \sim \epsilon^{2}$, so $B_{1}$ and $B_{2}$ tend to zero in approaching the line $p=2 \gamma$, maintaining relation $B_{1}^{2} \sim B_{2}$. For $\gamma<1$, the integral $\left(v_{0}, \tau^{2}\right)$ diverges and should be cut off at $\tau \sim 1 / A$, where transition to the logarithmic behavior (63) occurs; as a result $A \sim \epsilon^{1 /(2 \gamma-1)}, \delta u \sim \epsilon A \sim \epsilon^{2 \gamma /(2 \gamma-1)}$ 

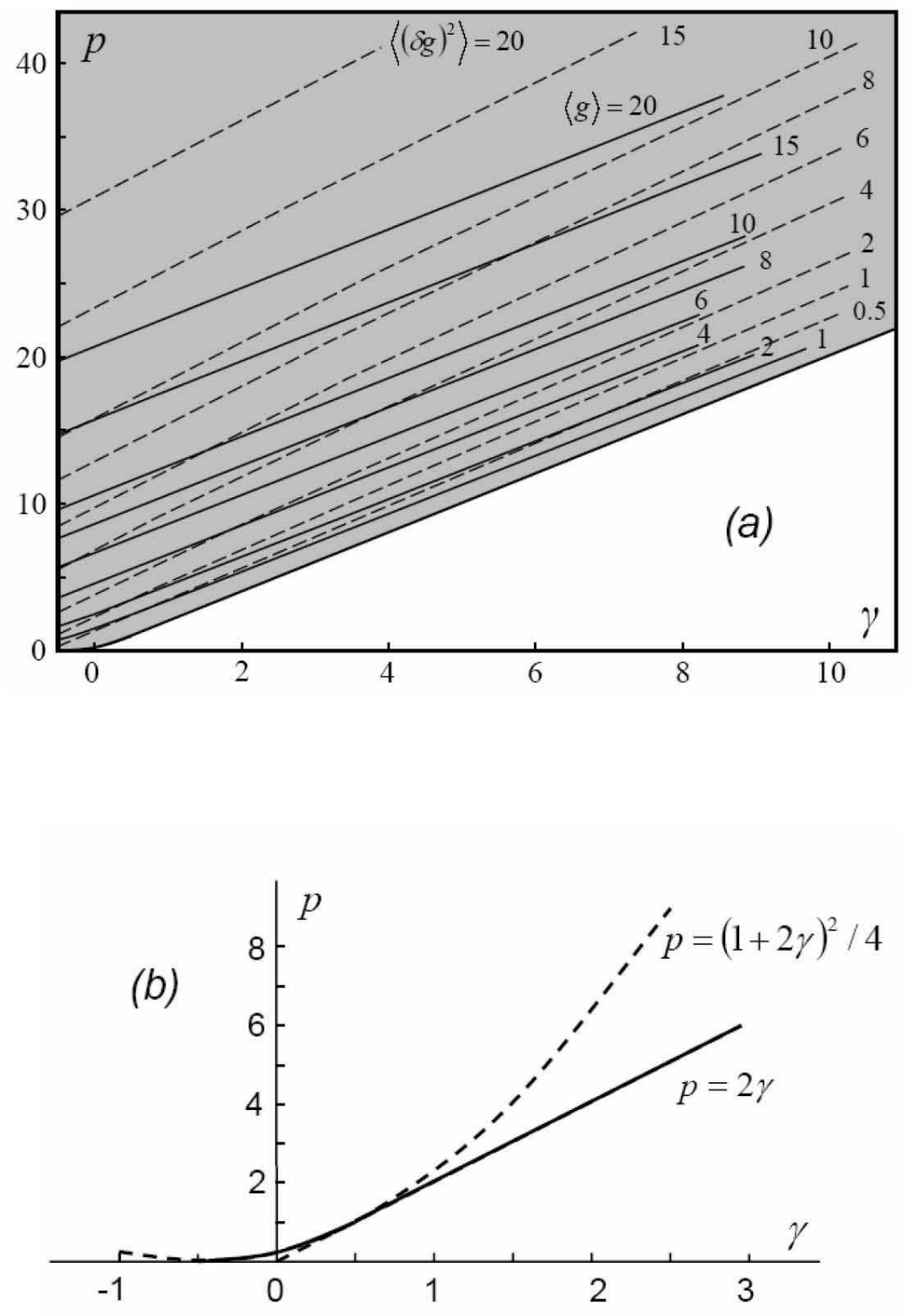

Figure 9: (a) The phase diagram for $\tau_{0}=1$. Solid and dashed lines correspond to constant values of $\langle g\rangle$ and $\left\langle(\delta g)^{2}\right\rangle$. (b) Construction, clarifying the arrangement of the lower boundary. 
and vanishing of the coefficients occurs under condition $B_{2} \sim B_{1}^{2 \gamma}$.

The line $p=(1+2 \gamma)^{2} / 4$ is distinguished due to the fact, that a linearized version of (48) reduces to the hypergeometric equation

$$
\tau(\tau+1) u^{\prime \prime}+(\alpha+\beta+1) \tau u^{\prime}+\alpha \beta u=0,
$$

where parameters $\alpha$ and $\beta$ are given by the formula

$$
\alpha, \beta=\frac{-(1+2 \gamma) \pm \sqrt{(1+2 \gamma)^{2}-4 p}}{2}
$$

and become coinciding at the indicated curve. A solution of (70), regular at the origin, has the following asymptotic regimes

$u(\tau)= \begin{cases}C\left[-\alpha \beta \tau+\frac{\alpha(\alpha+1) \beta(\beta+1)}{2} \tau^{2}+\ldots\right], & \tau \ll 1 \\ C\left[\frac{\Gamma(\alpha-\beta)}{\Gamma(\alpha) \Gamma(-\beta)} \tau^{-\beta}+\frac{\Gamma(\beta-\alpha)}{\Gamma(-\alpha) \Gamma(\beta)} \tau^{-\alpha}\right], & \tau \gg 1\end{cases}$

Coefficients of $\tau^{-\alpha}$ and $\tau^{-\beta}$ are determined by the matching conditions with (63), so the combinations $C \Gamma(\alpha-\beta)$ and $C \Gamma(\alpha-\beta)$ remain finite in the limit $\alpha \rightarrow \beta$. As a result, $C$ tends to zero due to divergency of the gamma functions, and the coefficients of $\tau$ and $\tau^{2}$ (i.e. $B_{1}$ and $B_{2}$ ) disappear according to the same law in approaching the curve $p=(1+2 \gamma)^{2} / 4$. The given arguments are correct under condition that matching with (63) occurs for sufficiently large $\tau$; practically this condition is fulfilled for $\gamma<0.5$.

\subsection{Metallic and dielectric regimes}

According to [3, 4, in the metallic state coefficients $B_{n}$ obey the same hierarchy $B_{1} \gg B_{2} \gg$ $B_{3} \gg \ldots$ as for $d=2+\epsilon$; the estimate $B_{n} \sim p / \tau_{0}^{n}$ follows from Eqs.50 for large $p$ and arbitrary $\gamma$, excluding a vicinity of the line $2 \gamma=p$. Substituting the arising representation $u(\tau)=p f\left(\tau / \tau_{0}\right)$ in the inverse Laplace transform, one can expand $f(x)$ in a series and retain two first terms; it leads to the Gaussian distribution. The case $2 \gamma \approx p$ can be considered separately and leads to the same conclusion (see Appendix 2).

The region of small $p$ can be analyzed rigorously for $\gamma=-1 / 2$. Linearizing (48) and omitting the small term $p u$, we have a solution

$$
u(\tau)=A \ln \left(1+\tau / \tau_{0}\right)
$$

tending to zero for $\tau \rightarrow 0$. If $A$ is sufficiently small, this solution remains valid in the region $\tau \gg \tau_{0}$, where $\tau\left(\tau+\tau_{0}\right)$ can be replaced by $\tau^{2}$. After it, equation (48) with $\gamma=-1 / 2$ has the exact solution

$$
u(x)=-\frac{1}{4} p\left(\ln \tau-x_{0}\right)^{2}+\frac{1}{2} .
$$

Within the accepted accuracy, one can replace $\tau$ by $\tau+\tau_{0}$ and provide the condition $u(0)=0$ by the appropriate choice of $x_{0}$. Then the solution

$$
u(x)=-(p / 4) \ln ^{2}\left(\tau+\tau_{0}\right)-\mu \ln \left(\tau+\tau_{0}\right)+b
$$

with arbitrary $\mu$ and $b=1 / 2-\mu^{2} / p, \tau_{0}=$ $\exp (\sqrt{2 / p}-2 \mu / p)$ is valid for all $\tau$; it is in agreement with (73) and for $\mu \ll 1$ provides a suggested smallness of $A$. Eq.75 corresponds to the log-normal distribution; it is evident for the saddle point calculation, but needs a more subtle analysis for small $p$; where applicability of the saddle-point method is strongly restricted (see Appendix 2). As demonstrated in the same Appendix, the approximately log-normal distribution remains valid for arbitrary values of $\gamma$ in the region of small $p$.

It is clear from above considerations, that large $p$ correspond to the metallic state, and small $p$ refer to the localized regime. Since parameters $p$ and $\gamma$ are in the one-to-one correspondence with $L / \xi$ and $L_{z} / L$, the certain line in the $(p, \gamma)$ plane corresponds to the cubical systems. This line originates at the point $p=0, \gamma=-1 / 2$ and goes to the region of large $p$.

\subsection{Critical region}

The critical region corresponds to values $p \sim 1$, when it is necessary to solve Eq.48 numerically, and numerically realize the inverse Laplace transformation. Integration of (48) begins at large $\tau$ in the logarithmic coordinates, starting from the asymptotics (63), and continues for $\tau \lesssim 1$ in the usual coordinates, adjusting $x_{0}$ to the condition $u(0)=0$. It is essential to control monotonicity of the solution, not allowing oscillations, which are possible according to Fig.7,b.

A numerical realization of the inverse Laplace transformation is conveniently made, using the rational approximation of $F(\tau)$ with subsequent decomposition to simple fractions

$$
F(\tau)=\frac{P_{M}(\tau)}{Q_{N}(\tau)}=\sum_{i=1}^{N} \frac{A_{i}}{\tau-\tau_{i}}
$$

where $P_{M}(\tau)$ and $Q_{N}(\tau)$ are polynomials of degree $M$ and $N$, and $M$ should be smaller than $N$ to 


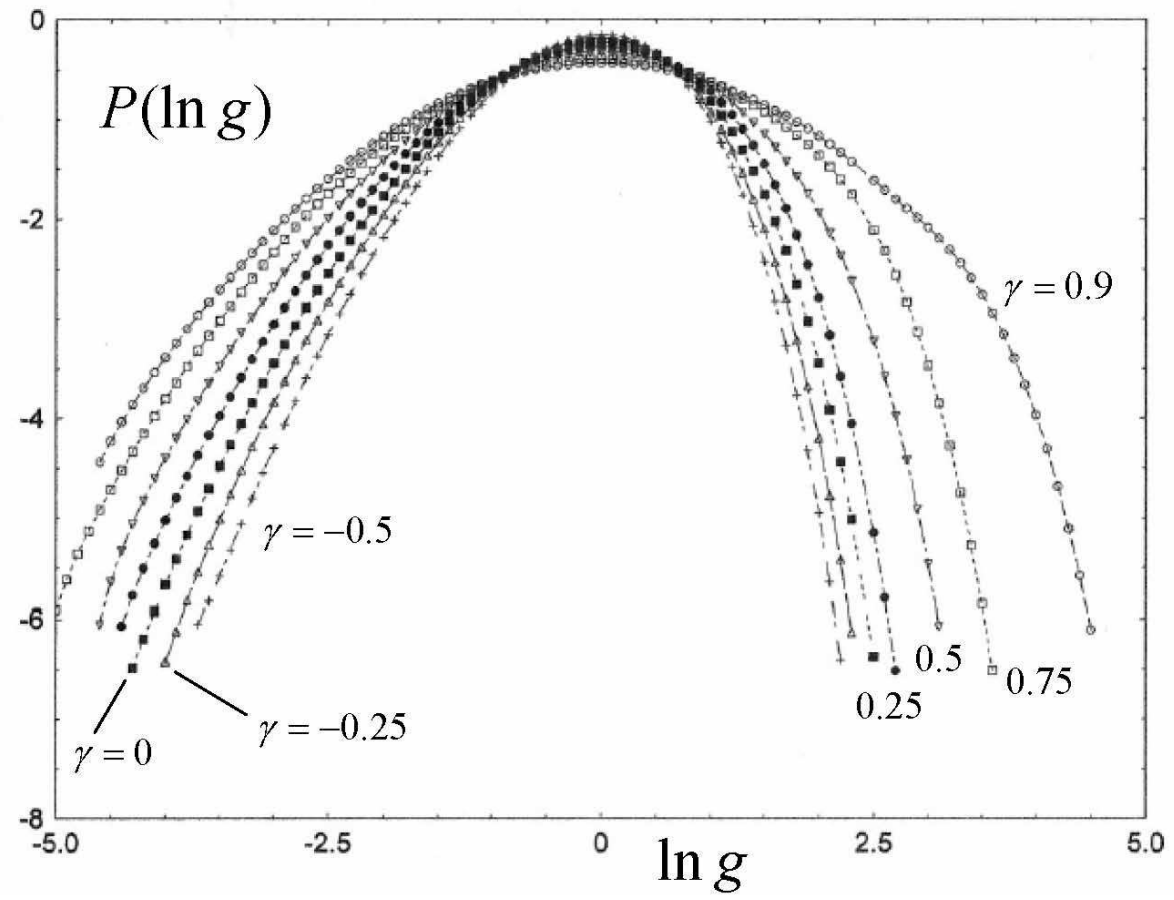

Figure 10: Distribution $P(\ln g)$ for different $\gamma$ and a fixed value $p=2$.

avoid $\delta$-functional contributions at the origin; after it, $W(g)$ is represented in the form

$$
W(g)=\sum_{i=1}^{N} A_{i} \exp \left\{\tau_{i} g\right\} .
$$

Due to decreasing of $W(g)$ for $g \rightarrow \infty$, the function $F(\tau)$ cannot contain singularities in the right half of the complex plane. In practice, the poles with a positive real part may arise due to "defects", manifested as pairs of the close pole and root: it leads to a catastrophic loss of accuracy or overflow. A practical recipe consists in the use of approximants of the maximal order, not containing the poles with a positive real part and providing the maximal accuracy of approximation for $F(\tau)$. A situation with "defects" can be optimized by changing the number and location of points on the $\tau$ axis, which are used for approximation (76).

Fig.10 demonstrates distributions $P(\ln g)$ for different $\gamma$ and a fixed value $p=2$. In a vicinity of the line $2 \gamma=p$, a distribution is practically symmetric and close to the log-normal one. With decreasing of $\gamma$, asymmetry of the distribution arises; at first it increases quickly, and then remains practically on the same level. Comparison with numerical data by Markos [11 is represented in Fig.11; agreement is satisfactory for parameters $\gamma=0, p=0.85$. Variation of the parameter $\tau_{0}$ does not change the form of the distribution $P(\ln g)$ and leads only to its parallel shift. For agreement with [11] one should set $\tau_{0}=0.67$, i.e. the choice $\tau_{0} \sim 1$ provides a correct scale of conductance in the critical region; it corresponds to expectations from the results for $d=2+\epsilon$.

As was indicated in the end of Sec.6.6, the trajectory corresponding to cubical systems exists in the $(p, \gamma)$ plane; along it the form of the distribution $P(\ln g)$ is modified due to a change of the amplitude of a random potential. A state of the system can be characterized by two parameters, a position on the trajectory and a value of $\tau_{0}$. In order the whole distribution obeys one-parameter scaling, it is sufficient and necessary that such scaling (i.e. dependence only on $L / \xi$ ) was valid for two independent parameters, characterizing this distribution, e.g. for $\langle g\rangle$ and $\left\langle(\delta g)^{2}\right\rangle$. Such property for the first parameter was established in [21] in the framework of selfconsistent theory of localization [22]; its validity for the second parameter looks rather probable due to results for the metallic $\left(\left\langle(\delta g)^{2}\right\rangle=\right.$ const $)$ and local- 
ized $\left(\left\langle(\delta g)^{2}\right\rangle \sim \exp (\right.$ const $\left.L / \xi)\right)$ phases.

According to Fig.11, a difference of the theore cal curve from the data by Markos 11 reduces smearing of a singularity at point $A$ (Fig.2). T difference is not surprising. In the present par we use the invariant definition of conductance, dependent of the way how the contact resistance the reservoir is excluded, and certainly character ing a finite system (Sec.4); in such situations sinধ larities are impossible in accordance with the $g_{t}$ eral principles [19, 20]. The definition used in [ is given by the many-channel Economou-Soukor formula [31, 32, which contains built-in singule ties: the distribution $W(g)$ for each channel drc off abruptly at $g=1$. This defect is related $\mathrm{w}$. the unsolved problem of the contact resistance and would be inessential in the true many-channel situation, when each channel carries a small part of conductance. In fact, the analysis by Markos shows [11, that the critical distribution is determined mainly by the most transparent channel, while the rest of channels forms only the exponential tail; so a deficiency of the definition is essential and directly leads to a singularity. On the other hand, absence in [11] of semi-transparent boundaries, separating the system from ideal leads, results in its strong interaction with the environment. The thermodynamic limit, realized by increasing the size of ideal leads, refers to the composite system "sample+ideal leads" and allows existence of singularities. In conclusion, a singularity at point $A$ is quite possible in the framework of the calculational scheme of [11, but is surely related with a deficient definition of conductance.

\section{Conclusion}

Above we have shown, that a modification of the Shapiro approach allows to introduce the twoparameter family of conductance distributions, defined by simple differential equations, whose parameters $p$ and $\gamma$ are in the one-to-one correspondence with parameters $L / \xi$ and $L_{z} / L$, characterizing a quasi-one-dimensional system. We did not try to calculate these parameters for any specific situations but investigate all family of distributions in whole. For large $p$ all distributions are Gaussian, which is typical for the metallic state. For small $p$ distributions are close to log-normal, in accordance with expectations for the localized phase. For $p \sim 1$ and the $\gamma$ values in the left part of the phase diagram, distributions are highly asymmetrical and close to the one-sided log-normal distribution, as established in

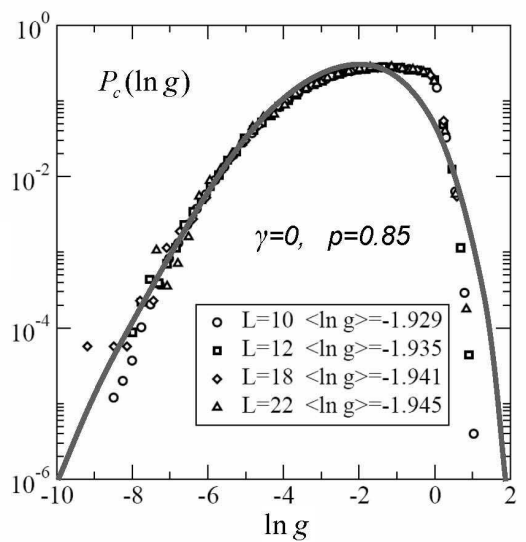

Figure 11: Comparison of numerical data by Markos [11] with the results of present theory for $\gamma=0, p=0.85$.

numerical experiments for the critical region. For a certain choice of parameters, we reproduce the results for cumulants $\left\langle\left\langle g^{n}\right\rangle\right\rangle$ in the space dimension $d=2+\epsilon$ obtained in the framework of the nonlinear $\sigma$-model approach. Numerical results for the critical distribution at $d=3$ are reproduced satisfactorily for $\gamma=0, p=0.85$ (Fig.11), excluding a singularity at point $A$ (Fig.2). The latter singularity is admissible in the framework of calculational scheme, used in [11, but related with a deficient definition of conductance.

The universal property of distributions is existence of two asymptotic regimes, log-normal for small $g$ and exponential for large $g$, while their actuality depends on a specific situation. In the metallic phase, a distribution is determined mainly by the central Gaussian peak, while two indicated asymptotic regimes refer to remote tails. In the critical region these two regimes determine practically all distribution, and the log-normal behavior extends to the region of the maximum. In the localized phase, the latter behavior extends even more and forces out the exponential asymptotics to the region of a remote tail.

The assumption of one-parameter scaling is the basis of the analysis, and its results are in agreement with this assumption. One-parameter scaling for the whole distribution takes place under condition, that two independent parameters characterizing this distribution, are functions of the ratio $L / \xi$. Such property is established in 21 for $\langle g\rangle$ and looks rather probable for $\left\langle(\delta g)^{2}\right\rangle$. 
The present paper fills in one of the main gaps in theory of disordered systems, related with absence of the systematic methods for investigation of distributions. Our conception, leading to Eq.47, is a natural consequence of one-parameter scaling and looks as completely justified; so the problem of the conductance distribution can be considered as solved in principle. The remaining free parameters can be fixed by calculation of the first several moments of conductance, which can be made by the standard methods.

\section{Appendix 1. Derivation of the evolution equation}

The increase of the length of a 1D system from $L$ till $L+\Delta L$ is assisted by multiplication of transfermatrices, $\hat{T}_{L+\Delta L}=\hat{T}_{L} \hat{T}_{\Delta L}$. Let assume a form (16) for the matrix $\hat{T}_{L}$ and use the following representation for the matrix $\hat{T}_{\Delta L}$

$$
\hat{T}_{\Delta L}=\left(\begin{array}{cc}
\sqrt{1+\epsilon^{2}} e^{i \beta_{1}} & -i \epsilon e^{i \beta_{2}} \\
i \epsilon e^{-i \beta_{2}} & \sqrt{1+\epsilon^{2}} e^{-i \beta_{1}}
\end{array}\right)
$$

where $\epsilon, \beta_{1}, \beta_{2}$ are small random quantities. The analogy with a point scatterer shows (see (26)), that $\epsilon$ is proportional to the amplitude of the random potential and its average should be set to zero, since in the other case it can be achieved by a change of the energy origin. Multiplying matrices, one obtains for the parameter $\tilde{\rho}$, corresponding to the matrix $\hat{T}_{L+\Delta L}$, in the second order in $\epsilon$

$$
\tilde{\rho}=\rho-2 \epsilon \sqrt{\rho(\rho+1)} \sin \psi+\epsilon^{2}(2 \rho+1) \equiv f(\rho),
$$

where

$$
\psi=\theta-\varphi+\beta_{1}+\beta_{2} .
$$

For the distribution of $\tilde{\rho}$ we have

$$
\begin{gathered}
P_{L+\Delta L}(\tilde{\rho})=\int d \rho d \psi d \epsilon P_{L}(\rho) P(\psi) P(\epsilon) \delta(\tilde{\rho}-f(\rho))= \\
=\int d \psi d \epsilon P(\psi) P(\epsilon) P_{L}\left(f_{1}(\tilde{\rho})\right) f_{1}^{\prime}(\tilde{\rho}), \quad(A .4)
\end{gathered}
$$

where $\rho=f_{1}(\tilde{\rho})$ is the inverse function to $\tilde{\rho}=f(\rho)$, which is found by iterations in $\epsilon$

$$
\begin{gathered}
f_{1}(\rho)=\rho+2 \epsilon \sqrt{\rho(\rho+1)} \sin \psi+ \\
+\epsilon^{2}(2 \rho+1)\left(2 \sin ^{2} \psi-1\right) .
\end{gathered}
$$

Substituting to $(A .4)$ and expanding to the second order in $\epsilon$, we have

$$
P_{L+\Delta L}(\rho)=P_{L}(\rho)\left[1+2 \overline{\epsilon^{2}}\left(2 \overline{\sin ^{2} \psi}-1\right)\right]+
$$

$$
\begin{gathered}
+P_{L}^{\prime}(\rho) \overline{\epsilon^{2}}(2 \rho+1)\left(4 \overline{\sin ^{2} \psi}-1\right)+ \\
+P_{L}^{\prime \prime}(\rho) \overline{\epsilon^{2}} \rho(\rho+1) 2 \overline{\sin ^{2} \psi},
\end{gathered}
$$

and setting $\overline{\epsilon^{2}}=\alpha \Delta L, \overline{\sin ^{2} \psi}=A_{0}$, we come to (14) with parameters (18), if small quantities $\beta_{1}$ and $\beta_{2}$ are neglected in $(A .3)$.

\section{Appendix 2. To investigation of Eq.48}

Let fill in the gaps in investigation of Eq.48 allowed in the main text.

$A$ vicinity of the line $p=2 \gamma$. In the case $p=$ $2 \gamma$, the linearized in $u$ equation (48) has an exact solution $u_{0}(\tau)=A \tau$ with small $A$, which is extended to the region of large $\tau$, where Eq.48 reduces to $(60)$ after the change $x=\ln \tau$. Considering $p \gg 1$ and retaining the main in $p$ terms, we have the equation

$$
u_{x}^{\prime 2}-p u_{x}^{\prime}+p u=0,
$$

whose solution can be written in the parametric form 33

$$
x=\ln \tau=t+\ln t+x_{0}, \quad u=-\frac{1}{4} p\left(t^{2}+2 t\right),
$$

where the running parameter $t$ changes from zero to infinity. Producing the inverse Laplace transformation and changing from integration over $\tau$ to integration over $t$, we have

$$
\begin{array}{r}
W(g)=\frac{1}{2 \pi i} \int d t(t+1) e^{t+x_{0}} . \\
\cdot \exp \left\{-\frac{1}{4} p\left(t^{2}+2 t\right)+g t e^{t+x_{0}}\right\},
\end{array}
$$

and the use of the saddle-point approximation leads to the log-normal distribution

$$
\begin{gathered}
W(g)=\sqrt{\frac{p}{4 \pi}} \frac{1}{g} \exp \left\{-\frac{p}{4}\left(\ln \frac{g_{c}}{g}\right)^{2}\right\}, \\
g_{c}=\frac{p}{2} e^{-x_{0}},
\end{gathered}
$$

reducing to the Gaussian one in the case of large $p$.

Small $p$. For $\gamma=-1 / 2$, the solution $u(\tau)$ is determined by Eq.75, whose substitution to the inverse Laplace transform and subsequent changes $\tau \rightarrow \tau-\tau_{0}$ and $\tau \rightarrow \tau / g$ give

$$
W(g)=\frac{1}{2 \pi i} g^{-1+\mu} \int_{-i \infty}^{i \infty} d \tau \tau^{-\mu} e^{\tau} .
$$




$$
\cdot \exp \left\{-\frac{p}{4} \ln ^{2} \tau+\frac{p}{2} \ln \tau \ln g-\frac{p}{4} \ln ^{2} g+b-\tau_{0} g\right\} .
$$

Expanding the exponent in $p \ln \tau$ and calculating the integrals for small $\mu$, we have

$$
W(g) \sim \frac{1}{g} \exp \left\{-\frac{p}{4}\left(\ln g-\frac{2 \mu}{p}+\frac{1}{\mu}\right)^{2}-\tau_{0} g\right\} .
$$

and the term $\tau_{0} g$ can be omitted for $\mu \ll \sqrt{p}$. Consideration is valid for $p|\ln g| \ll 1$, which in the case $p \ll \mu \ll \sqrt{p}$ covers a vicinity of the maximum and describes all essential part of the distribution.

In fact, the approximately log-normal distribution is valid in the region of small $p$ for the arbitrary value of $\gamma$. Indeed, setting $\gamma=-1 / 2+\epsilon$ (where $0 \leq$ $\epsilon \leq \sqrt{p}$ ) and omitting the term $p u$ in the linearized equation (48), we have the solution

$$
u(\tau)=C_{1}\left(\tau+\tau_{0}\right)^{2 \epsilon}+C_{2},
$$

which reduces to (73) for $\epsilon \ln \left(\tau+\tau_{0}\right) \ll 1$. On the other hand, Eq.60 with $\tilde{\gamma}=2 \epsilon$ has an approximate solution for large $x$

$u(x)=-\frac{1}{4} p x^{2}+\epsilon x(\ln x-1)-\left(\epsilon^{2} / p\right) \ln ^{2} x+\frac{1}{2}+O\left(x^{-1}\right)$, (A.14)

where $O\left(x^{-1}\right)$ contains terms of type $\ln ^{m} x / x$. Neglecting slow variation of $\ln x$ and replacing it by a suitable constant, one can see that invariance relative $x \rightarrow x-x_{0}$ allows to reduce (A.14) to a form (75).

\section{References}

[1] B. L. Altshuler, JETP Lett. 41, 648 (1985) [Pis'ma Zh. Eksp. Teor. Fiz.41, 530 (1985)];

B. L. Altshuler, D. E. Khmelnitskii, JETP Lett. 42, 359 (1985) [Pis'ma Zh. Eksp. Teor. Fiz. 42, $291(1985)]$.

[2] P. A. Lee, A. D. Stone, Phys. Rev. Lett. 55, 1622 (1985);

P. A. Lee, A. D. Stone, Y. Fukuyama, Phys. Rev. B 35, 1039 (1987).

[3] B. L. Altshuler, V. E. Kravtsov, I. V. Lerner, Sov. Phys. JETP 64, 1352 (1986) [Zh. Eksp. Teor. Fiz. 91, 2276 (1986)];

[4] B. L. Altshuler, V. E. Kravtsov, I. V. Lerner Phys. Lett. A 134, 488 (1989).
[5] E. Abrahams, P. W. Anderson, D. C. Licciardello, and T. V. Ramakrishman, Phys. Rev. Lett. 42, 673 (1979).

[6] B. Shapiro, Phys. Rev. B 34, 4394 (1986).

[7] B. Shapiro, Phil. Mag. 56, 1031 (1987).

[8] B. Shapiro, Phys. Rev. Lett. 65, 1510 (1990).

[9] I. M. Suslov, Sov. Phys. JETP 50, 499 (1987) [Zh. Eksp. Teor. Fiz. 92, 1433 (1987)].

[10] M. Rühländer, C. M. Soukoulis, Physica B Cond Matt. 296, 32 (2001).

[11] P. Markoš, acta physica slovaca 56, 561 (2006).

[12] K. A. Muttalib, P. Wölfle, A. Garcia-Martin, V. A. Gopar, Europhys. Lett. 61, 95 (2003).

[13] K. Slevin, T. Ohtsuki, P. Markoš, Phys. Rev. B 67, 155106 (2003).

[14] K. Slevin, T. Ohtsuki, P. Markoš, Phys. Rev. Lett. 86, 3594 (2001).

[15] V. I. Melnikov, Sov. Phys. Sol. St. 23, 444 (1981) [Fizika Tverdogo Tela 23, 782 (1981)].

[16] N. Kumar, Phys. Rev. B 31, 5513 (1985).

[17] P. Mello, Phys. Rev. B 35, 1082 (1987).

[18] A. A. Abrikosov, Sol. St. Comm. 37, 997 (1981).

[19] K. Wilson and J. Kogut, Renormalization Group and the Epsilon Expansion (Wiley, New York, 1974).

[20] S. Ma, Modern Theory of Critical Phenomena (Benjamin, Reading, Mass., 1976).

[21] I. M. Suslov, JETP 115, 897 (2012) [Zh. Eksp. Teor. Fiz. 142, 1020 (2012)]

[22] D. Vollhardt, P. Wölfle, Phys. Rev. B 22, 4666 (1980); Phys. Rev. Lett. 48, 699 (1982).

[23] I. M. Lifshitz, V. Ya. Kirpichenkov, Sov. Phys. JETP 50, 499 (1979) [Zh. Eksp. Teor. Fiz. 77, 989 (1979)].

[24] B. Shapiro, E. Abrahams, Phys. Rev. B 24, 4889 (1981).

[25] I. M. Suslov, JETP 113, 619 (2011) [Zh. Eksp. Teor. Fiz. 140, 712 (2011)]; arXiv:1506.06128. 
[26] A. Pelissetto, E. Vicari, Phys. Rep. 368, 549 (2002), Sec.2.3.

[27] P. W. Anderson, D. J. Thouless, E. Abrahams, D. S. Fisher, Phys. Rev. B 22, 3519 (1980).

[28] R. Landauer, IBM J. Res. Dev. 1, 223 (1957); Phil. Mag. 21, 863 (1970).

[29] M. Kappus, F. Wegner, Z. Phys. B 45, 15 (1981).

V. E. Kravtsov, V. I. Yudson, Ann. Phys. (NY) 326, 1672 (2011).

[30] A. Cohen, Y. Roth, B. Shapiro, Phys. Rev. B 38, 12125 (1988).

[31] E. N. Economou, C. M. Soukoulis, Phys. Rev. Lett. 46, 618 (1981).

[32] A. D. Stone, A. Szafer, IBM J. Res. Dev. 32, 384 (1988).

[33] E. Kamke, Handbuch on the Ordinary Differential Equations, Moscow, Nauka, 1976. 